
\title{
An Explosive Instability of Kelvin-Helmholtz Flows in Ferrofluids: Effect of Periodic Tangential Magnetic Field With Rotation
}

\author{
P.T. Hemamalini* \\ Department of Science and Humanities, Karpagam Academy of Higher Education, \\ Coimbatore -641021, Tamil Nadu, India; shanthi2saba@gmail.com
}

\begin{abstract}
Objectives: The work is to learn the impingement of tangential periodic magnetic field on the interface in the middle of two superposed ferrofluids with the rotation through a nonlinear perturbation analysis. Methods or Statistical Analysis: A nonlinear instability due to streaming superposed ferrofluids stressed by rotation and a periodic tangential magnetic field is examined by employing the mode of multiple scales. The fluids are taken to be incompressible. Using the boundary conditions, the solutions of the linearized equations of motion lead to the linear dispersion equation. Findings: From this work, it is clearly seen the unfolding of amplitude of waves was influenced by an equation of nonlinear Schrodinger. Applications or Improvements: The rotation and a periodic tangential magnetic field have enormous important effects over the stability of the system.
\end{abstract}

Keywords: Dispersion Relation and Schrodinger Equation, Ferrofluids, Linear Dispersion Relation, Method of Multiple Scales, Rotation

\section{Introduction}

Ferrofluids (called magnetic fluids) are stable colloidal cessation of magnetic nanoparticles. Brownian motion maintains the particles from settling and their surface is coated with a dispersant in order to stop them from sticking together. An applied magnetic field yields a new external parameter that can be stabilize or destabilize the fluid interface producing hydrodynamic instabilities. The liquid carrier in commericially produced ferrofluids are kerosene, toluene, water or one of several other fluids.

The ferromagnetic particles are typically $30-150 \mathrm{~A}$ in diameter and are magnetite, iron or cobalt. The electrical conductivity of a ferrofluid was in the same order as that of the liquid carrier, which is typically quite small. The Brownian motion also keeps the tiny magnets randomly oriented so the ferrofluid has no natural magnetic moment. Magnetic fluids are used in vacuum which seals around the rotating shafts and in loudspeakers to keep them cool and to damp vibrations. Experiments of mag- netic fluids with rotation and magnetic field have shown in a wide range of interesting phenomena.

RTI happens when a weighty fluid is assisted by a light-weight fluid. Because of the wide applications of the RTI in planetary and steller atmospheres etc, several studies have been addressed. Kelvin-Helmholtz Instability (KHI) arises when fluids are in a relative motion.

The hydrodynamic stability for RTI, KHI and other has been reported through the pioneer book of $\frac{1-3}{\underline{ }}$ investigated the interfacial stability of a ferromagnetic fluid. An excellent book on the stability theory is given by $y^{ \pm}$. They reported the analysis of RHI, KHI and capillary instability of perfect fluids. The linear KHI of flow in porous media is studied by ${ }^{5}$. He derived the instability conditions in KHI for Darcian and non-Darcian flows. Non linear stability of surface waves in magnetic fluids with tangential periodic magnetic field was analyzed by ${ }^{6}$. Nonlinear KHI of two miscible ferrofluids in porous media was investigated $b y^{ }$. He analyzed linear as well as nonlinear aspects of stability theory of the interface in middle of two super-

${ }^{*}$ Author for correspondence 
posed magnetic fluids. Kelvin-Helmholtz instabilities in smoothed particle hydrodynamics have been treated extensively by ${ }^{\circ}$. KHI in a fluid layer bounded by a porous layer above and below by a rigid surface in the presence of magnetic field was discussed by?

$\mathrm{In}^{10}$ experimented the stability analysis on KelvinHelmholtz flow of an electrified horizontal sheet through porous media investigated the instability of KelvinHelmholtz flows in magnetic fluids have examined the nonlinear KHI of superposed ferrofluids under rotation and magnetic field $\frac{11-13}{}$.

\section{Fluid-field Configuration}

Let us imagine a heavy fluid $\left(\rho_{1}\right)$ supported by a light fluid $\left(\rho_{2}\right)$ under the gravity. The interface in between the two fluids is described as $z=\eta(x, t)$. From the undisturbed position, the elevation of the wave is measured and is denoted by $\eta(x, t)$. When the interface is completely flat then $z=\eta(x, t)=0$. A fluid with density $\left(\rho_{1}\right)$ and permeability $\left(\mu_{1}\right)$ hold the region $z<0$ whereas $z>0$ is occupied by a fluid density $\left(\rho_{2}\right)$, magnetic permeability $\left(\mu_{2}\right)$. Here Cartesian coordinate system is chosen. It is assumed that $\mathrm{X}$-axis rest in the unperturbed interface. $\mathrm{Z}$-axis is taken perpendicular which is pointed from the lower level fluid to the upper fluid. The fluids are taken to be inviscid magnetic fluids moving with uniform velocity $U_{1}$ and $U_{2}$ in the $x$-direction and the fluid is assumed to rotate in the uniform angular velocity $\Omega$ about $Z$-axis. The physical quantities are undertake to differ with respect to $x$ and $z$ coordinates except ' $t$ ', since the disturbances are constricted to two dimensional plane waves. The system is emphasized by a tangential periodic magnetic field $\boldsymbol{H}=\varepsilon^{\frac{1}{2}} H_{0} \cos \omega t \bar{i}$ in the direction of X-axis. Here $\varepsilon$ denotes the small non-dimensional parameter, $H_{0}$ shows the magnetic field amplitude, $\omega$ marks the field frequency and along the direction of $\mathrm{X}$-axis the unit vector is taken as $\bar{i}$.

Analysis begins with the basic equations, the NavierStokes equation:

$$
\rho \frac{d \boldsymbol{q}}{d t}+2 \rho(\Omega \times q)=-\nabla p+(\dot{A}+\delta \dot{A}) \boldsymbol{g}+\mu_{0} \boldsymbol{M} \cdot \nabla \boldsymbol{H}
$$

Here $\boldsymbol{q}=(u+U, v, w), \Omega=(0,0, \Omega)$, the final term $\mu_{0} \boldsymbol{M} . \nabla \boldsymbol{H}$ describes the magnetic body force on the fluid element, $\boldsymbol{M}$ is the magnetization vector and $\propto_{0}$ takes the value 1 .
From a potential function, assume that an additional magnetic field ' $h$ ' is derived.

i.e. $\psi(x, z)$ exists with $h=-\varepsilon^{\frac{1}{2}} \nabla \psi$.

The magnetic potential $\psi$ satisfies the equation:

$$
\begin{aligned}
& \nabla^{2} \psi^{(1)}=0, \quad-\infty<z<\eta(x, t) \\
& \nabla^{2} \psi^{(2)}=0, \quad \eta(x, t)<z<\infty
\end{aligned}
$$

The kinematic boundary condition is defined at $z$ is equal to $\eta(x, t)$.

$\frac{\partial \eta}{\partial t}+\left(u^{i}+U^{i}\right) \frac{\partial \eta}{\partial x}-w^{i}=0, \mathrm{i}=1,2$.

Here $\mathrm{u}$ represents the tangential velocity and $\mathrm{w}$ indicates the normal velocity. $U^{1}, U^{2}$ represents the speed of the fluid in region (1) and (2) respectively.

Non viscous fluid satisfies $\hat{n} \cdot \boldsymbol{q}^{(1)}=\hat{n} \cdot \boldsymbol{q}^{(2)}$

Continuity of normal and tangential components of the magnetic field at:

$$
\begin{aligned}
& \quad z=h(x, t), \text { are } \\
& \mu_{1} H_{n}^{(l)}=\mu_{2} H_{n}^{(2)} \\
& H_{t}^{(l)}=H_{t}^{(2)}
\end{aligned}
$$

Normal stress is balanced on the system at the dividing surface.

$$
\left[\left[-p+\frac{\mu}{2}\left(H_{n}^{2}-H_{t}^{2}\right)\right]\right]=\frac{-T \eta_{x x}}{\left(1+\eta_{x}^{2}\right)^{\frac{3}{2}}} \text { at } z=h(x, t)
$$

Where $p$ indicates the magnetic strictive pressure, $T$ shows the coefficient of the surface tension, [[]] signifying the jump in the amount across the regions 1 and 2.

\section{Perturbation Methodology}

By applying the mode of multiple scales, the perturbed equations are solved by taking $\varepsilon$ which represents the steepness ratio of the wave. The values of $t_{n}$ and $x_{n}$ interpreted as:

$$
t_{n}=t \frac{1}{\varepsilon^{-n}}, x_{n}=x \frac{1}{\varepsilon^{-n}} \quad(n=0 \text { to } 2)
$$


velocity.

where $\varepsilon=\left(\frac{U}{U_{m}}-1\right)^{\frac{1}{2}}$ and $U_{m}$ denotes the marginal

In order to examine the transition of a carrier wave with wave number $k$, frequency $\omega$, pretend the dependence of $\eta(x, t)$ to be:

$\eta(x, t)=\sum_{n=1}^{3} \varepsilon^{n} \eta_{n}\left(x_{0}, x_{1}, x_{2} ; t_{0}, t_{1}, t_{2}\right)+O\left(\varepsilon^{4}\right)$

and $\eta_{1}=A\left(x_{1}, x_{2} ; t_{1}, t_{2}\right) \exp i\left(k x_{0}-\omega_{0} t_{0}\right)+C . C$

Here C.C represents the complex conjugate of all previous terms and $A$ marks amplitude of the propagating wave which is slowly varying function. $k$ denotes the wave number, $\omega_{0}$ marks the frequency of the disturbance.

Expand the physical quantities $q$ and $\psi$ as,

$\boldsymbol{q}(x, z, t)=\sum_{n=1}^{3} \varepsilon^{n} \boldsymbol{q}_{n}\left(x_{0}, x_{1}, x_{2}, z ; t_{0}, t_{1}, t_{2}\right)+O\left(\varepsilon^{4}\right)$

$\psi(x, z, t)=\sum_{n=1}^{3} \varepsilon^{n} \psi_{n}\left(x_{0}, x_{1}, x_{2}, z ; t_{0}, t_{1}, t_{2}\right)+O\left(\varepsilon^{4}\right)$

The scale $x_{0}$ stands for the wavelength; the scale $t_{0}$ marks the frequency of the wave. The scales of the phase, amplitude are denoted by $t_{1}$ and $t_{2}$ respectively whereas spatial modulations of the phase is denoted by $x_{1}$ and the amplitude is noted by $x_{2}$

Utility the series of Maclaurins at $z=0$ for the quantities, the boundary conditions are evaluated. Replacing the above equations (9) - (13) into a series (1) - (8) and comparing the particulars of same powers of $\varepsilon, \varepsilon^{2}, \varepsilon^{3}$ etc, to reach 3 consequent equations.

\section{Linear Dispersion Relation}

By using first order equations, we arrive at the linear dispersion relation:

$$
\begin{aligned}
\rho_{2}\left(U_{2} k-\omega_{0}\right)^{2} & \frac{1}{\left(1-\frac{4 \Omega^{2}}{\left(U_{2} k-\omega_{0}\right)^{2}}\right)^{-\frac{1}{2}}}+ \\
& \rho_{1}\left(U_{1} k-\omega_{0}\right)^{2} \frac{1}{\left(1-\frac{4 \Omega^{2}}{\left(U_{1} k-\omega_{0}\right)^{2}}\right)^{-\frac{1}{2}}}
\end{aligned}
$$

$$
=\left(\rho_{1}-\rho_{2}\right) g k-\alpha^{2} k^{2}+T k^{3}
$$

$$
\text { Where } \alpha^{2}=\frac{H_{0}^{2} \cos ^{2} \omega t(1-\mu)^{2} \mu_{2}}{(1+\mu)} \text { and } \mu=\frac{\mu_{1}}{\mu_{2}}
$$

The dispersion relation (14) coincides with the result of a linear perturbation 1 in the absence of both rotation, magnetic field for RTI. In addition, if there is no field frequency, then to learn the amplitude modulation of the progressive waves, we assume $\omega_{0}^{2}>0$. The system is stable if $\left(\rho_{1}-\rho_{2}\right) g-\alpha^{2} k+T k^{2}>0$.

\section{Second Order Problem}

The solution of the linear order is substituted into the equations of second order. Solve them by assuming,

$$
\begin{aligned}
\eta_{2}= & \wedge A^{2} \exp (2 i \theta)+C . C \\
\text { Where } \wedge=\frac{N r}{D r} & \\
N r= & -H_{0}^{2} \cos ^{2} \omega t k^{2} \mu_{2}(1-\mu)\left(\frac{(1-\mu)}{(1+\mu)}\right)^{2} \\
& +\frac{2}{k^{2}}\left[\rho_{2} \gamma_{2} \beta_{2}\left(\omega_{0}-U_{2} k\right)^{2}-\rho_{1} \gamma_{1} \beta_{1}\left(\omega_{0}-U_{1} k\right)^{2}\right] \\
& -\frac{2 \Omega^{2}}{k^{2}}\left(\rho_{2} \gamma_{2} \beta_{2}-\rho_{1} \gamma_{1} \beta_{1}\right)+ \\
& \frac{1}{k^{2}}\left[\rho_{1} \beta_{1}^{2}\left(\omega_{0}-U_{1} k\right)^{2}-\rho_{2} \beta_{2}^{2}\left(\omega_{0}-U_{2} k\right)^{2}\right] \\
& +\frac{4 \Omega^{2}}{k^{2}}\left(\rho_{2} \beta_{2}^{2}-\rho_{1} \beta_{1}^{2}\right) \\
D r & =-\frac{2}{k^{2}}\left[\rho_{2} \gamma_{2}\left(\omega_{0}-U_{2} k\right)^{2}+\rho_{1} \gamma_{1}\left(\omega_{0}-U_{1} k\right)^{2}\right] \\
& +2 k H_{0}^{2} \cos ^{2} \omega t \frac{(1-\mu)^{2} \mu_{2}}{(1+\mu)}+\frac{2 \Omega^{2}}{k^{2}}\left(\rho_{2} \gamma_{2}+\rho_{1} \gamma_{1}\right) \\
& -\left(\rho_{2}-\rho_{1}\right) g+4 k^{2} T
\end{aligned}
$$

Where

$$
\begin{aligned}
& \beta_{1}=k\left(1-\frac{4 \Omega^{2}}{\left(U_{1} k-\omega_{0}\right)^{2}}\right)^{-\frac{1}{2}} \\
& \beta_{2}=k\left(1-\frac{4 \Omega^{2}}{\left(U_{2} k-\omega_{0}\right)^{2}}\right)^{-\frac{1}{2}}
\end{aligned}
$$




$$
\begin{aligned}
& \gamma_{1}=k\left(1-\frac{\Omega^{2}}{\left(U_{1} k-\omega_{0}\right)^{2}}\right)^{-\frac{1}{2}} \\
& \gamma_{2}=k\left(1-\frac{\Omega^{2}}{\left(U_{2} k-\omega_{0}\right)^{2}}\right)^{-\frac{1}{2}}
\end{aligned}
$$

\section{Third Order Problem}

The dispersion relation of third order is:

$$
\begin{aligned}
& \frac{\partial^{2} A}{\partial x_{1}^{2}}\left\{\frac{\rho_{2} \omega_{0}}{k^{3}}\left(\frac{\beta_{2} \omega_{0}}{k}+\frac{s_{3}}{2 \beta_{2}}\right)+\frac{\rho_{1} \omega_{0}}{k^{3}}\left(\frac{\beta_{1} \omega_{0}}{k}+\frac{s_{1}}{2 \beta_{1}}\right)+\frac{\rho_{2} U_{2} \beta_{2}}{k^{3}}\left(\omega_{0}-U_{2} k\right)+\frac{\rho_{1} U_{1} \beta_{1}}{k^{3}}\left(\omega_{0}-U_{1} k\right)-\frac{\rho_{2} U_{2}}{k^{2}}\left(\frac{\beta_{2} \omega_{0}}{k}+\frac{s_{3}}{2 \beta_{2}}\right)\right. \\
& -\frac{\rho_{1} U_{1}}{k^{2}}\left(\frac{\beta_{1} \omega_{0}}{k}+\frac{s_{1}}{2 \beta_{1}}\right)+\frac{4 \Omega^{2} \rho_{2}}{k^{3}\left(\omega_{0}-U_{2} k\right)}\left(U_{2} \beta_{2}-\frac{\beta_{2} \omega_{0}}{k}-\frac{s_{3}}{2 \beta_{2}}\right)-\frac{4 \Omega^{2} \rho_{1}}{k^{3}\left(\omega_{0}-U_{1} k\right)}\left(-U_{1} \beta_{1}+\frac{\beta_{1} \omega_{0}}{k}+\frac{s_{1}}{2 \beta_{1}}\right)-\frac{4 \Omega^{2} \rho_{2}}{k^{4}} \beta_{2}-\frac{4 \rho_{1} \Omega^{2}}{k^{4}} \beta_{1} \\
& +\frac{\rho_{2}}{k^{4}} \beta_{2}\left(\omega_{0}-U_{2} k\right)^{2}+\frac{\rho_{1}}{k^{4}} \beta_{1}\left(\omega_{0}-U_{1} k\right)^{2}+\frac{\omega_{0} \rho_{2}}{k^{3}}\left(\frac{\beta_{2} \omega_{0}}{k}+\frac{s_{3}}{2 \beta_{2}}\right)+\frac{\omega_{0} \rho_{1}}{k^{3}}\left(\frac{\beta_{1} \omega_{0}}{k}+\frac{s_{1}}{2 \beta_{1}}\right)-\frac{4 \Omega^{2} \rho_{2}}{k^{3}\left(\omega_{0}-U_{2} k\right)}\left(\frac{\beta_{2} \omega_{0}}{k}+\frac{s_{3}}{2 \beta_{2}}\right) \\
& \left.-\frac{4 \Omega^{2} \rho_{1}}{k^{3}\left(\omega_{0}-U_{1} k\right)}\left(\frac{\beta_{1} \omega_{0}}{k}+\frac{s_{1}}{2 \beta_{1}}\right)-\frac{4 U_{2} \Omega^{2} \rho_{2}}{k^{2}\left(\omega_{0}-U_{2} k\right)^{2}}\left(U_{2} \beta_{2}-\frac{\beta_{2} \omega_{0}}{k}-\frac{s_{3}}{2 \beta_{2}}\right)+\frac{4 U_{1} \Omega^{2} \rho_{1}}{k^{2}\left(\omega_{0}-U_{1} k\right)^{2}}\left(-U_{1} \beta_{1}+\frac{\beta_{1} \omega_{0}}{k}+\frac{s_{1}}{2 \beta_{1}}\right)-\right\} \\
& +\frac{\partial^{2} A}{\partial x_{1} \partial t_{1}}\left\{\frac{\rho_{2} \beta_{2}}{k^{3}}\left(\omega_{0}-U_{2} k\right)+\frac{\rho_{1} \beta_{1}}{k^{3}}\left(\omega_{0}-U_{1} k\right)+\frac{2 \rho_{2} \omega_{0}}{k^{3}}\left(\beta_{2}+\frac{s_{4}}{2 \beta_{2}}\right)+\frac{2 \rho_{1} \omega_{0}}{k^{3}}\left(\beta_{1}+\frac{s_{2}}{2 \beta_{1}}\right)-\frac{\rho_{2} U_{2}}{k^{2}}\left(\beta_{2}+\frac{s_{4}}{2 \beta_{2}}\right)-\frac{\rho_{1} U_{1}}{k^{2}}\left(\beta_{1}+\frac{s_{2}}{2 \beta_{1}}\right)\right. \\
& -\frac{2 \Omega^{2} \rho_{2} s_{4}}{k^{3} \beta_{2}\left(\omega_{0}-U_{2} k\right)}-\frac{2 \Omega^{2} \rho_{1} s_{2}}{k^{3} \beta_{1}\left(\omega_{0}-U_{1} k\right)}+\frac{\rho_{2}}{k^{2}}\left(\frac{\beta_{2} \omega_{0}}{k}+\frac{s_{3}}{2 \beta_{2}}\right)+\frac{\rho_{1}}{k^{2}}\left(\frac{\beta_{1} \omega_{0}}{k}+\frac{s_{1}}{2 \beta_{1}}\right) \\
& -\frac{2 \Omega \rho_{2}}{k\left(\omega_{0}-U_{2} k\right)}\left[\frac{2 \Omega}{k^{2}}\left[\beta_{2}+\frac{s_{4}}{2 \beta_{2}}\right]+\frac{2 \Omega}{k\left(\omega_{0}-U_{2} k\right)}\left[U_{2} \beta_{2}-\frac{\beta_{2} \omega_{0}}{k}-\frac{s_{3}}{2 \beta_{2}}\right]-\frac{U_{2} \Omega s_{4}}{\beta_{2} k\left(\omega_{0}-U_{2} k\right)}\right]+\frac{2 \Omega \rho_{1}}{k\left(\omega_{0}-U_{1} k\right)}\left[-\frac{2 \Omega}{k^{2}}\left[\beta_{1}+\frac{s_{2}}{2 \beta_{1}}\right]\right. \\
& \left.+\frac{2 \Omega}{k\left(\omega_{0}-U_{1} k\right)}\left[\left[-U_{1} \beta_{1}+\frac{\beta_{1} \omega_{0}}{k}+\frac{s_{1}}{2 \beta_{1}}\right]+\frac{U_{1} \Omega s_{2}}{\beta_{1} k\left(\omega_{0}-U_{1} k\right)}\right]\right\}+\frac{\partial A}{\partial x_{2}}\left\{\left(1-\mu_{2}\right) H_{0}^{2} \cos ^{2} \omega t i\left(\frac{1-\mu}{1+\mu}\right)-\left(1-\mu_{1}\right) H_{0}^{2} \cos ^{2} \omega t i\left(\frac{1-\mu}{1+\mu}\right)\right. \\
& +\frac{8 \rho_{2} \Omega^{2} i}{k^{3}} \beta_{2}+\frac{8 \rho_{1} \Omega^{2} i}{k^{3}} \beta_{1}-\frac{\rho_{2} \beta_{2} i}{k^{3}}\left(\omega_{0}-U_{2} k\right)^{2}-\frac{\rho_{1} \beta_{1} i}{k^{3}}\left(\omega_{0}-U_{1} k\right)^{2}-\frac{\rho_{2} \beta_{2} \omega_{0}}{k^{2}}\left(\omega_{0}-U_{2} k\right)-\frac{\rho_{1} \beta_{1} \omega_{0}}{k^{2}}\left(\omega_{0}-U_{1} k\right) \\
& \left.+\frac{\rho_{2} \beta_{2} U_{2}}{k}\left(\omega_{0}-U_{2} k\right)+\frac{\rho_{1} \beta_{1} U_{1}}{k}\left(\omega_{0}-U_{1} k\right)-\frac{i \rho_{2} \beta_{2} U_{2}}{k^{2}}\left(\omega_{0}-U_{2} k\right)-\frac{i \rho_{1} \beta_{1} U_{1}}{k^{2}}\left(\omega_{0}-U_{1} k\right)-2 T i k\right\} \\
& +\frac{\partial^{2} A}{\partial t_{1}^{2}}\left\{\frac{\rho_{2}}{k^{2}}\left[\beta_{2}+\frac{s_{4}}{2 \beta_{2}}\right]+\frac{\rho_{1}}{k^{2}}\left[\beta_{1}+\frac{s_{2}}{2 \beta_{1}}\right]+\frac{2 \Omega^{2} \rho_{2} s_{4}}{k^{2}\left(\omega_{0}-U_{2} k\right)^{2} \beta_{2}}+\frac{2 \Omega^{2} \rho_{1} s_{2}}{k^{2}\left(\omega_{0}-U_{1} k\right)^{2} \beta_{1}}\right\}
\end{aligned}
$$




$$
\begin{aligned}
& -\frac{\partial A}{\partial t_{2}}\left\{\frac{\rho_{2} \beta_{2} i}{k^{2}}\left(\omega_{0}-U_{2} k\right)+\frac{\rho_{1} \beta_{1} i}{k^{2}}\left(\omega_{0}-U_{1} k\right)\right\}+A^{2} \bar{A}\left\{\frac{H_{0}^{2} \cos ^{2} \omega t k^{2}(1-\mu)}{2(1+\mu)^{2}}\left(\mu_{2}-1\right)[k(\mu+3)+(1-\mu) 6 \Lambda+4 \Lambda \mu]-\right. \\
& \frac{H_{0}^{2} \cos ^{2} \omega t k^{2}(1-\mu)}{2(1+\mu)^{2}}\left(\mu_{1}-1\right)[k(\mu+3)+(1-\mu) 6 \Lambda-4 \Lambda] \\
& +4 k^{2}\left(\mu_{2}-1\right) H_{0}^{2} \cos ^{2} \omega t\left(\frac{1-\mu}{1+\mu}\right)^{2}(\Lambda+k)-4 k^{2}\left(\mu_{1}-1\right) H_{0}^{2} \cos ^{2} \omega t\left(\frac{1-\mu}{1+\mu}\right)^{2}(\Lambda-k) \\
& -\frac{2 \rho_{2} \beta_{2} \gamma_{2}}{k^{2}}\left(\omega_{0}-U_{2} k\right)^{2}\left(\beta_{2}+\Lambda\right)-\frac{2 \rho_{1} \beta_{1} \gamma_{1}}{k^{2}}\left(\omega_{0}-U_{1} k\right)^{2}\left(\beta_{1}-\Lambda\right)-\frac{4 \rho_{2} \gamma_{2}^{2}}{k^{2}}\left(\omega_{0}-U_{2} k\right)^{2}\left(\beta_{2}+\Lambda\right) \\
& -\frac{4 \rho_{1} \gamma_{1}^{2}}{k^{2}}\left(\omega_{0}-U_{2} k\right)^{2}\left(\beta_{1}-\Lambda\right)+\frac{2 \rho_{2} \beta_{2}^{2}}{k^{2}}\left(\omega_{0}-U_{2} k\right)^{2}\left(\Lambda+\beta_{2}\right)+\frac{2 \rho_{1} \beta_{1}^{2}}{k^{2}}\left(\omega_{0}-U_{1} k\right)^{2}\left(\beta_{1}-\Lambda\right) \\
& +\frac{4 \omega_{0}}{k^{2}}\left(\rho_{2} \gamma_{2}^{2}\left(\omega_{0}-U_{2} k\right)\left(\Lambda+\beta_{2}\right)+\rho_{1} \gamma_{1}^{2}\left(\omega_{0}-U_{2} k\right)\left(\beta_{1}-\Lambda\right)\right) \\
& -\frac{4}{k}\left(\rho_{2} U_{2} \gamma_{2}^{2}\left(\omega_{0}-U_{2} k\right)\left(\beta_{2}+\Lambda\right)+\rho_{1} U_{1} \gamma_{1}^{2}\left(\omega_{0}-U_{1} k\right)\left(\beta_{1}-\Lambda\right)\right) \\
& -\frac{4 \Omega^{2}}{k^{2}}\left(\rho_{2} \gamma_{2}^{2}\left(\beta_{2}+\Lambda\right)-\rho_{1} \gamma_{1}^{2}\left(\beta_{1}-\Lambda\right)\right)-4 H_{0}^{2} \cos ^{2} \omega t k^{2}\left(\frac{1-\mu}{1+\mu}\right)\left(\left(\mu_{2}-1\right)(\Lambda+k)+\left(1-\mu_{1}\right)(k-\Lambda)\right) \\
& \left(\mu_{2}-1\right) H_{0}^{2} \cos ^{2} \omega t k^{3}\left(\frac{1-\mu}{1+\mu}\right)-\left(\mu_{1}-1\right) H_{0}^{2} \cos ^{2} \omega t k^{3}\left(\frac{1-\mu}{1+\mu}\right)+\frac{4 \Omega^{2}}{k^{2}}\left(\rho_{2} \beta_{2}^{3}-\rho_{1} \beta_{1}^{3}\right) \\
& -\frac{1}{k^{2}}\left(\rho_{2} \beta_{2}^{3}\left(\omega_{0}-U_{2} k\right)^{2}-\rho_{1} \beta_{1}^{3}\left(\omega_{0}-U_{1} k\right)^{2}\right)-4 H_{0}^{2} \cos ^{2} \omega t k^{3}\left(\frac{1-\mu}{1+\mu}\right)\left(\mu_{2}-\mu_{1}\right) \\
& +H_{0}^{2} \cos ^{2} \omega t k^{3}\left(\frac{1-\mu}{1+\mu}\right)^{2}\left(2 \mu_{1}+2 \mu_{2}-1\right)+4 H_{0}^{2} \cos ^{2} \omega t k^{2} \Lambda\left(\frac{1-\mu}{1+\mu}\right)\left(\mu_{1}+\mu_{2}\right) \\
& -4 H_{0}^{2} \cos ^{2} \omega t k^{2}\left(\frac{1-\mu}{1+\mu}\right)\left(2 \Lambda-\mu_{2}(\Lambda+k)+\mu_{1}(\Lambda-k)\right) \\
& +\left(\mu_{1}-1\right) H_{0}^{2} \cos ^{2} \omega t i\left(\frac{1-\mu}{1+\mu}\right)+\frac{4 \rho_{2} \Omega^{2} i}{k^{3}} \beta_{2}-\rho_{2} \frac{\left(\omega_{0}-U_{2} k\right)^{2} i \beta_{2}}{k^{3}}-\rho_{1} \frac{\left(\omega_{0}-U_{1} k\right)^{2} i \beta_{1}}{k^{3}}+4 H_{0}^{2} \cos ^{2} \omega t k^{2} \Lambda\left(\frac{1-\mu}{1+\mu}\right)\left(\mu_{1}+\mu_{2}\right) \\
& -4 H_{0}^{2} \cos ^{2} \omega t k^{2}\left(\frac{1-\mu}{1+\mu}\right)^{2}\left(\mu_{2}(k+\Lambda)+\mu_{1}(k-\Lambda)-k\right)-4 H_{0}^{2} \cos ^{2} \omega t k^{2} \Lambda\left(\mu_{2}-\mu_{1}\right) \\
& \left.+4 H_{0}^{2} \cos ^{2} \omega t k^{3}\left(\frac{1-\mu}{1+\mu}\right)^{2}\left(\mu_{1}+\mu_{2}-1\right)-2 H_{0}^{2} \cos ^{2} \omega t k^{3}\left(\frac{1-\mu}{1+\mu}\right)^{2}\left(\mu_{2}-\mu_{1}\right)-\frac{3}{2} k^{4} T\right\}=0 \\
& \text { Where } s_{1}=U_{1}\left[k^{2}+\beta_{1}^{2}-\frac{4 \Omega^{2} \beta_{1}^{2}}{\left(U_{1} k-\omega_{0}\right)^{2}}\right]-\frac{2 \beta_{1}^{2}}{k}\left[\omega_{0}+\frac{4 \Omega^{2}}{\left(U_{1} k-\omega_{0}\right)}\right] \\
& s_{2}=k^{2}-\beta_{1}^{2}-\frac{4 \Omega^{2} \beta_{1}^{2}}{\left(U_{1} k-\omega_{0}\right)^{2}}, s_{3}=U_{2}\left[k^{2}+\beta_{2}^{2}-\frac{4 \Omega^{2} \beta_{2}^{2}}{\left(U_{2} k-\omega_{0}\right)^{2}}\right]-\frac{2 \beta_{2}^{2}}{k}\left[\omega_{0}+\frac{4 \Omega^{2}}{\left(U_{2} k-\omega_{0}\right)}\right] \\
& s_{4}=k^{2}-\beta_{2}^{2}-\frac{4 \Omega^{2} \beta_{2}^{2}}{\left(U_{2} k-\omega_{0}\right)^{2}}
\end{aligned}
$$




\section{Nonlinear Schrodinger Equation and Stability Conditions}

Let the coefficients of the terms $\frac{\partial^{2} A}{\partial x_{1}^{2}}, \frac{\partial^{2} A}{\partial x_{1} \partial t_{1}}, \frac{\partial^{2} A}{\partial t_{1}^{2}}$, $\frac{\partial A}{\partial x_{2}}, \frac{\partial A}{\partial t_{2}}$ in Equation (18) are represented by $N_{1 k}^{*}, N_{2 k}^{*}$, $N_{3 k}{ }^{*}, N_{4 k}{ }^{*}$ and $N_{5 k}{ }^{*}$ respectively.

Third order dispersion relation presumes the form:

$i \frac{\partial A}{\partial \tau}+\beta_{1 k}^{*} \frac{\partial^{2} A}{\partial \xi^{2}}+\beta_{k}^{*} \frac{\partial A}{\partial \xi}+Q_{k}|A|^{2} A=0$

Which is the well-known second order nonlinear Schrodinger equation.

Where:

$\beta_{1 k}^{*}=\frac{i}{N_{5 k}}\left[N_{1 k}-C_{g k} N_{2 k}+C_{g k}^{2} N_{3 k}\right]$

$\beta_{k}^{*}=i\left(\frac{N_{4 k}}{N_{5 k}}-C_{g k}\right)$

$Q_{k}=\frac{i}{N_{5 k}} Q_{1 k}$ Where $Q_{1 k}=\frac{\partial D}{\partial|A|^{2}}$ and $C_{g k}=-\frac{D k}{D \omega_{0}}$ with

$$
\begin{array}{rl}
D k= & \frac{\rho_{2}}{2}\left[\left(U_{2} k-\omega_{0}\right)^{4}-4 \Omega^{2}\left(U_{2} k-\omega_{0}\right)^{2}\right]^{-\frac{1}{2}} \\
& {\left[4 U_{2}\left(U_{2} k-\omega_{0}\right)^{3}-8 \Omega^{2} U_{2}\left(U_{2} k-\omega_{0}\right)\right]} \\
+ & \frac{\rho_{1}}{2}\left[\left(U_{1} k-\omega_{0}\right)^{4}-4 \Omega^{2}\left(U_{1} k-\omega_{0}\right)^{2}\right]^{-\frac{1}{2}} \\
& {\left[4 U_{1}\left(U_{1} k-\omega_{0}\right)^{3}-8 \Omega^{2} U_{1}\left(U_{1} k-\omega_{0}\right)\right]} \\
-\left(\rho_{1}-\rho_{2}\right) g+2 H_{0}^{2} \cos ^{2} \omega t k \frac{(1-\mu)^{2} \mu_{2}}{(1+\mu)}-3 T k^{2} & 2 \rho_{2} \\
D \omega_{0}= & \frac{2 \rho_{1}}{\left(\left(U_{2} k-\omega_{0}\right)^{2}-4 \Omega^{2}\right)^{\frac{1}{2}}}\left[2 \Omega^{2}-\left(U_{2} k-\omega_{0}\right)^{2}\right] \\
& +\frac{\left(\left(U_{1} k-\omega_{0}\right)^{2}-4 \Omega^{2}\right)^{\frac{1}{2}}}{\left(2 \Omega^{2}-\left(U_{1} k-\omega_{0}\right)^{2}\right]}
\end{array}
$$

Which are obtained from linear dispersion equation.
The stability criterion is analyzed, using ${ }^{6}$ by considering the following time varying solution.

$A=m e^{i Q_{k} m^{2} \tau}, m$ is a constant.

Perturb the Equation (20) by stating:

$A=\left[m+D_{1 k}(\xi, \tau)+i E_{1 k}(\xi, \tau)\right] e^{i Q_{k} m^{2} \tau}$

Where $D_{1 k}$ and $E_{1 k}$ are real functions.

Using Equation (21) in Equation (19) and neglecting the terms which are nonlinear in $D_{1 k}$ and $E_{1 k}$.

$-\frac{\partial E_{1 k}}{\partial \tau}+\beta_{1 k}^{*} \frac{\partial^{2} D_{1 k}}{\partial \xi^{2}}+\beta_{k}^{*} \frac{\partial D_{1 k}}{\partial \xi}=0$ and

$\frac{\partial D_{1 k}}{\partial \tau}+\beta_{1 k}^{*} \frac{\partial^{2} E_{1 k}}{\partial \xi^{2}}+\beta_{k}^{*} \frac{\partial E_{1 k}}{\partial \xi}=0$

Writing

$D_{1 k}(\xi, \tau)=D^{*} e^{\left(i k_{2} \xi+\omega_{2 k} \tau\right)}+C . C$

and $E_{1 k}(\xi, \tau)=E^{*} e^{\left(i k_{2} \xi+\omega_{2 k} \tau\right)}+C . C$

where $D^{*}$ and $E^{*}$ are constant values, $k_{2}$ denotes the wave number and $\omega_{2 k}$ indicates the disturbance frequency. By using (24) and (25), $\omega_{2 k}$ and $k_{2}$ desire the dispersion relation.

$\omega_{2 k}^{2}+\left[\beta_{1 k}^{* 2} k_{2}^{4}-k_{2}^{2} \beta_{k}^{* 2}-2 i k_{2}^{3} \beta_{k}^{*} \beta_{1 k}^{*}\right]=0$

where the wave number $k_{2}$ may assume real and positive values.

which implies

$\omega_{2 k}= \pm\left(J_{R}+i J_{I}\right)$

where $J_{R}=\left(\frac{G_{R}+\sqrt{\left(G_{R}^{2}+G_{I}^{2}\right)}}{2}\right)^{\frac{1}{2}}$ and

$J_{I}=\frac{G_{I}}{\left|G_{I}\right|}\left(\frac{\left.-G_{R}+\sqrt{\left(G_{R}^{2}+G_{I}^{2}\right.}\right)}{2}\right)^{\frac{1}{2}}$

$G_{R}=C_{1}^{2} k_{2}^{2}-D_{1}^{2} k_{2}^{2}-\beta_{1 k}^{* 2} k_{2}^{4}-2 \beta_{1 k}^{*} D_{1} k_{2}^{3}$

$G_{I}=2 C_{1} D_{1} k_{2}^{2}+2 C_{1} \beta_{1 k}^{*} k_{2}^{3}$

$D_{1}=\left(\frac{B_{1}}{A_{2}}-C_{g k}\right)$ 


$$
\begin{aligned}
C_{1}= & \frac{1}{\rho_{2} \beta_{2}\left(U_{2} k-\omega_{0}\right)+\rho_{1} \beta_{1}\left(U_{1} k-\omega_{0}\right)}\left[\left(U_{2} k-\omega_{0}\right)\right. \\
& \rho_{2} \beta_{2} \omega_{0}+\left(U_{1} k-\omega_{0}\right) \rho_{1} \beta_{1} \omega_{0}-\left(U_{1} k-\omega_{0}\right) \rho_{1} \beta_{1} U_{1} k \\
& \left.-\left(U_{2} k-\omega_{0}\right) \rho_{2} \beta_{2} U_{2} k\right] \\
A_{2}= & \frac{\rho_{1} \beta_{1}}{k^{2}}\left(U_{1} k-\omega_{0}\right)+\frac{\rho_{2} \beta_{2}}{k^{2}}\left(U_{2} k-\omega_{0}\right) \\
B_{1}= & \left(1-\mu_{2}\right) H_{0}^{2} \cos ^{2} \omega t\left(\frac{(1-\mu)}{(1+\mu)}\right) \\
& -\left(1-\mu_{1}\right) H_{0}^{2} \cos ^{2} \omega t\left(\frac{(1-\mu)}{(1+\mu)}\right) \\
& +8 \rho_{2} \Omega^{2} \frac{\beta_{2}}{k^{3}}+8 \rho_{1} \Omega^{2} \frac{\beta_{1}}{k^{3}} \\
& -\frac{1}{k^{3}}\left(\left(U_{2} k-\omega_{0}\right)^{2} \beta_{2} \rho_{2}+\left(U_{1} k-\omega_{0}\right)^{2} \beta_{1} \rho_{1}\right) \\
& +\frac{1}{k^{2}}\left(\rho_{2} U_{2} \beta_{2}\left(U_{2} k-\omega_{0}\right)+\rho_{1} U_{1} \beta_{1}\left(U_{1} k-\omega_{0}\right)\right)-2 T K
\end{aligned}
$$

\section{Discussion and Conclusion}

Linear dispersion relation (14) coincides with Rosensweig in the absence of rotation, the horizontal magnetic field. It also coincides with Chandrasekhar in the non-appearance of rotation, magnetic field.

It is clearly seen from the above values that the system is found to be unstable when:

$$
\begin{aligned}
& J_{R}=\left(\frac{G_{R}+\sqrt{\left(G_{R}^{2}+G_{I}^{2}\right)}}{2}\right)^{\frac{1}{2}}>0, \\
& J_{R}=\left(\frac{G_{R}+\sqrt{\left(G_{R}^{2}+G_{I}^{2}\right)}}{2}\right)^{\frac{1}{2}}<0 \text { and } \\
& \left(U_{1} k-\omega_{0}\right)^{2}>4 \Omega^{2},\left(U_{2} k-\omega_{0}\right)^{2}>4 \Omega^{2} .
\end{aligned}
$$

Hence it is concluded that rotation and a periodic tangential magnetic field have great significant effects over the stability of the system.

\section{References}

1. Chandrasekhar S. Hydrodynamic and hydromagnetic stability. Oxford University Press: London; 1961.

2. Rosensweig RE. Ferro hydrodynamics. Cambridge University Press; 1985.

3. Cowley MD, Rosensweig RE. The interfacial stability of a ferromagnetic fluid. Journal of Fluid Mechanics. 1967; 30:671-88. https://doi.org/10.1017/S0022112067001697.

4. Drazin P. Hydrodynamic stability. Cambridge Univ Press; 1981.

5. Bau HH. Kelvin-Helmholtz instability of parallel flow in porous media: A linear theory. Phys of Fluids. 1982; 25:1719-22. https://doi.org/10.1063/1.863642.

6. El-Dib YO. Non linear stability of surface waves in magnetic fluids: Effect of a periodic tangential magnetic field. Journal of Plasma Physics. 1993; 49:317-30. https://doi.org/10.1017/S0022377800017013.

7. Moatimid GM. Nonlinear KHI of two miscible ferro fluids in porus media. ZAMP. 2006; 57:133-59. https://doi. org/10.1007/s00033-005-2067-1.

8. Valcke S, De Rijcke S, Rodiger E, Dejonghe H. KHI In smoothed particle hydrodynamics. Monthly Notices of the Royal Astronomical Society. 2010; 408:71-86. https://doi.org/10.1111/j.1365-2966.2010.17127.x.

9. Chavaraddi B. KHI instability in a fluid layer bounded by a porous layer above and by a rigid surface below in presence of magnetic field. Applied Mathematics. 2012; 3:564-70. https://doi.org/10.4236/am.2012.36086.

10. Moatimid GM, El-dabe NT. Sayed A. Stability analysis of a Kelvin-Helmholtz flow of an electrified horizontal sheet through porous media. Journal of Scientific and Engineering Research. 2018; 5:119-35.

11. Khosla HK, Malik SK. Non linear explosive instability of Kelvin-Helmholtz flows in magnetic fluids. Journal of Magnetism and Magnetic Materials. 1996; 164:390-400. https://doi.org/10.1016/S0304-8853(96)00400-3.

12. Hemamalini PT, Anjali Devi SP. Rayleigh-Taylor instability of a two fluid layer subjected to rotation and a periodic tangential magnetic field. FDMP. 2014; 10:491-501.

13. Weissmann MA. Nonlinear wave packets in the KHI, Philos. Transactions of the Royal Society A. 1979; 290:63985. https://doi.org/10.1098/rsta.1979.0019. 